\title{
Dynamics of Momentary Reserves under Contingency: Observations from Numerical Experiments
}

\author{
Kosisochukwu Pal Nnoli \\ Dept. of Computer Science and Electrical Engineering
Jacobs University, 28759 Bremen \\ Bremen, Germany \\ k.nnoli@jacobs-university.de
}

\author{
Stefan Kettemann \\ Dept. of Physics and Earth Science and Dept. of Computer Science \\ Jacobs University, 28759 Bremen \\ Bremen, Germany \\ Division of Advanced Materials Science, Pohang 790-784 \\ South Korea \\ s.kettemann@jacobs-university.de
}

\begin{abstract}
This paper presents the studies and investigations on the dynamics of momentary reserves in electrical power systems under contingency. Momentarily reserve through the machine's inertia serves the purpose of primary frequency control and prevents voltage collapse in the case of reactive power reserves. A simulation was performed on a realistic Nigerian $330 \mathrm{kV}$ transmission network in PowerFactory software to study and investigate the mechanism of these reserve functions on the network buses as an inertia active power control method. Moreover, we investigated the influence of geodesic increment of momentary reserve on the decay of disturbances. The results indicated that the momentary reserve by inertia alone reduces the frequency deviation from its nominal value, delays the transmission of disturbances and enhances the damping of oscillations by reducing the final frequency settling time at the buses under contingency. This numerical experiment also suggests the optimal placement of the momentary reserves in the grid in order to improve system stability against power outage disturbances.

Index Terms-momentary reserve, system disturbance, inertia control, oscillations damping, frequency stability.
\end{abstract}

\section{INTRODUCTION}

The active and reactive power reserves of synchronous generators or battery energy storage systems (BESS) are the keys to a successful system control in power systems. For each power generator in the grid, power reserve represents the total amount of power remaining after the supply of system loads and losses. Of course, this definition does not extend to exceeding the power capability curve of the generators. These reserves are particularly referred to as spinning reserves in synchronous machines more than in other kinds of power generators, like the wind and solar generators using BESS [1]. These reserves can be used for both or either primary frequency control, secondary control and tertiary control [2] [3] [4]. Again, not all of these remaining power reserves from generators are assigned for primary control function alone, we classify the ones momentarily made available through

Bundesministerium für Bildung und Forschung (BMBF) CoNDyNet-2, FK. 03EK3055D. the generators' droop functions specifically for few seconds primary frequency and active power control as momentarily reserves of the generators. A lot about system stability and reliability rest on the grid's momentarily reserve.

The control and response of generators to network disturbances or contingencies depends heavily on the kind and magnitude of the disturbance and amount of momentary reserves available for use in the primary frequency control or in voltage security. Since primary control requires fast control action to be taken within few seconds of contingency, momentary reserves and their placement play important roles in the dynamics of the disturbance from the event location to the rest of the electrical power network. In this paper, we investigate how the dynamics of a disturbance is influenced by momentary reserves first at the contingent node, its neighbour nodes and other nodes located far away from the fault location. We would see whether a disturbance is damped as it travels across a grid and whether it could be contained on fewer nodes (i.e. localized) based on the function of the grid's momentarily reserves. These investigations are carried out in the DigSILENT PowerFactory software [5], using the Nigerian $330 \mathrm{kV}$ grid as a case study.

\section{Modeling and Simulation of the Nigerian TRANSMISSION NETWORK MODEL}

To understand our case study system and its parameter interactions, we would describe the components that make up the network. The Nigerian $330 \mathrm{kV}$ transmission grid consist of $N_{S}=71$ substations/nodes, $N_{L}=81$ over-head transmission lines (from an alloy of aluminium and steel) with an average length of $92 \mathrm{Km}$, each with a limiting current of $1320 \mathrm{~A}$. The grid is comprised of 107 less-decommissioned units of generators, accounting for the present 29 power stations. The apparent power capacity of the Nigerian grid is about $13,208 M W$ as of 2020 [6]. There are other lower voltage networks including the $132 \mathrm{kV}$ and $33 \mathrm{kV}$ sub-transmission networks. For household utilities, there are $11 \mathrm{kV}$ and 0.415 
$\mathrm{kV}$ 3-phase distribution networks. The Nigerian network operates at $\nu_{0}=50 \mathrm{~Hz}$ frequency and can be described as a grid where most of the nodes are connected to one another in a ring form [7], as seen in the diagram shown in Fig.1.

To control the voltage outputs of the generators through their excitation control, the simplified excitation automatic voltage regulator (AVR) model is used [8]. Other controllers also include power system stabilizers (PSS2A-model) tasked with enhancing the damping of the entire power system's oscillations through excitation control.

The input signal to the PSS2A controller is the derivative of generator's rotor speed injected to the AVR through the excitation system. This injection works to terminate the phase-lag between the voltage reference and the windings' torque of the generator [9]. For the synchronous machine model, the choice of the model is influenced by the IEEE guide in [10]. The speed governor in the model is the turbine governor (TGOV) model used to maintain the frequency operational limits according to swing equation [11]. Here, the swing equation describes the torque balance between the mechanical torque $T_{t}$ in N.m. of each synchronous machine's turbine and the electromagnetic torque $T_{e}$ in N.m. as governed by the differential equation given as [2] [3] [12],

$$
J_{i} \frac{d \omega_{i}^{\Theta}}{d t}+D_{r_{i}} \omega_{i}^{\Theta}=T_{t}-T_{e}-D_{r_{i}} \omega_{0}
$$

where $J_{i}=\frac{2 H_{i}}{\omega_{0}^{2}} S_{i}$ is the combined moment of inertia of the generator and turbine in $\mathrm{kg} \cdot \mathrm{m}^{2}$ with $H_{i}$ the generator inertia constant in seconds and $S_{i}$ the generator apparent power in volt-amperes $(V A) . D_{r_{i}}$ represents the rotational loss due to generator rotor windings for each $i^{\text {th }}$ generator in N.m.s and $i$ denotes the index of power generators in the grid. Here, $t$ is time in seconds and $\omega_{i}^{\Theta}$ is the angular velocity of the rotor in electrical $\mathrm{rad} / \mathrm{s}$ with $\omega_{0}$ as its rated synchronous value in electrical $\mathrm{rad} / \mathrm{s}$. If we assume that a change in the rotor's angular velocity $\left(\omega_{i}^{\Theta}-\omega_{0}\right)$ is a derivative of its angular position $\delta$ in electrical radians with respect to its rotating setpoint, $\delta_{0}$ at $\mathrm{t}=0$ given as

$$
\omega_{i}^{\Theta}-\omega_{0}=\frac{d \delta_{i}}{d t}
$$

and with respect to time, the derivative of $\omega_{i}^{\Theta}$ would give

$$
\frac{d \omega_{i}^{\Theta}}{d t}=\frac{d}{d t}\left(\frac{d \delta_{i}}{d t}\right)+\frac{d \omega_{0}}{d t},
$$

where $\omega_{0}$ is the constant rated synchronous value whose derivative with respect to time gives zero (i.e. $\frac{d \omega_{0}}{d t}=0$ ), Eq.(3) becomes,

$$
\frac{d \omega_{i}^{\Theta}}{d t}=\frac{d^{2} \delta}{d t^{2}}
$$

In practice, $\omega_{0}$ is related to the grid frequency $\left(v_{o}\right)$ by $2 \pi v_{o}$, where $v_{o}$ is $50 \mathrm{~Hz}$ in the Nigerian power grid. If we represent the net mechanical shaft torque at grid frequency to be $T_{m}=$ $T_{t}-D_{r_{i}} \omega_{0}$, substituting Eq.(4) into Eq.(1), we then have,

$$
J_{i} \frac{d^{2} \delta_{i}}{d t^{2}}+D_{r_{i}}\left(\frac{d \delta_{i}}{d t}\right)=T_{m}-T_{e}
$$

Here, we assumed that the network perturbation effected on the rotors from the fault location is small. Multiplying both sides of Eq. $(5)$ by the rated speed $\left(\omega_{0}\right)$ in order to ensure that we maintain a synchronous $50 \mathrm{~Hz}$ revolution throughout the system, balancing the power, we have

$$
J_{i} \omega_{0} \frac{d^{2} \delta_{i}}{d t^{2}}+D_{r_{i}} \omega_{0}\left(\frac{d \delta_{i}}{d t}\right)=T_{m} \omega_{0}-T_{e} \omega_{0}
$$

As power $P=T \omega$, the right side of Eq.(6) can now be written as

$$
J_{i} \omega_{0} \frac{d^{2} \delta_{i}}{d t^{2}}+D_{r_{i}} \omega_{0}\left(\frac{d \delta_{i}}{d t}\right)=P_{m}-P_{e}
$$

where $P_{m}$ is the turbine's mechanical power and $P_{e}$ is the generator's air-gap electrical power. If we represent the rotor angular momentum at rated speed with $M_{i}$ (i.e. $M_{i}=J_{i} \omega_{0}=$ $\frac{2 H_{i}}{\omega_{0}} S_{i}$ ) and also represent the damping coefficient at rated synchronous speed with $D_{i}$ (i.e. $D_{i}=D_{r_{i}} \omega_{0}$ ), the swing equation can then be re-written in many forms as,

$$
M_{i} \frac{d^{2} \delta_{i}}{d t^{2}}+D_{i}\left(\frac{d \delta_{i}}{d t}\right)=P_{m}-P_{e}
$$

and also as [13] [14],

$$
\frac{2 H_{i}}{\omega_{o}} S_{i} \frac{d^{2} \delta_{i}}{d t^{2}}+D_{i} \frac{d \delta_{i}}{d t}=P_{i}+\sum_{j=1}^{N_{S}} W_{i j} \sin \left(\delta_{j}-\delta_{i}\right)
$$

Where $P_{i}$ is the power in the grid's $i^{\text {th }}$ node, $N_{S}$ is the number of nodes/buses and $W_{i j}$ is the power capacity in Watt of the transmission lines and it is dependent on the network voltage with $\sin \left(\delta_{j}-\delta_{i}\right)$ modeling the dependence of their phase differences which informs the direction of powerflow and the transmission of disturbances in the case of contingencies. We performed load-flow calculations using the Newton-Raphson method and electromechanical simulations in DigSILENT PowerFactory software, as documented in Ref. [15]. Here, we report results applying these simulations to study the effect of momentary reserves on system dynamics and its contribution to the overall system stability. 


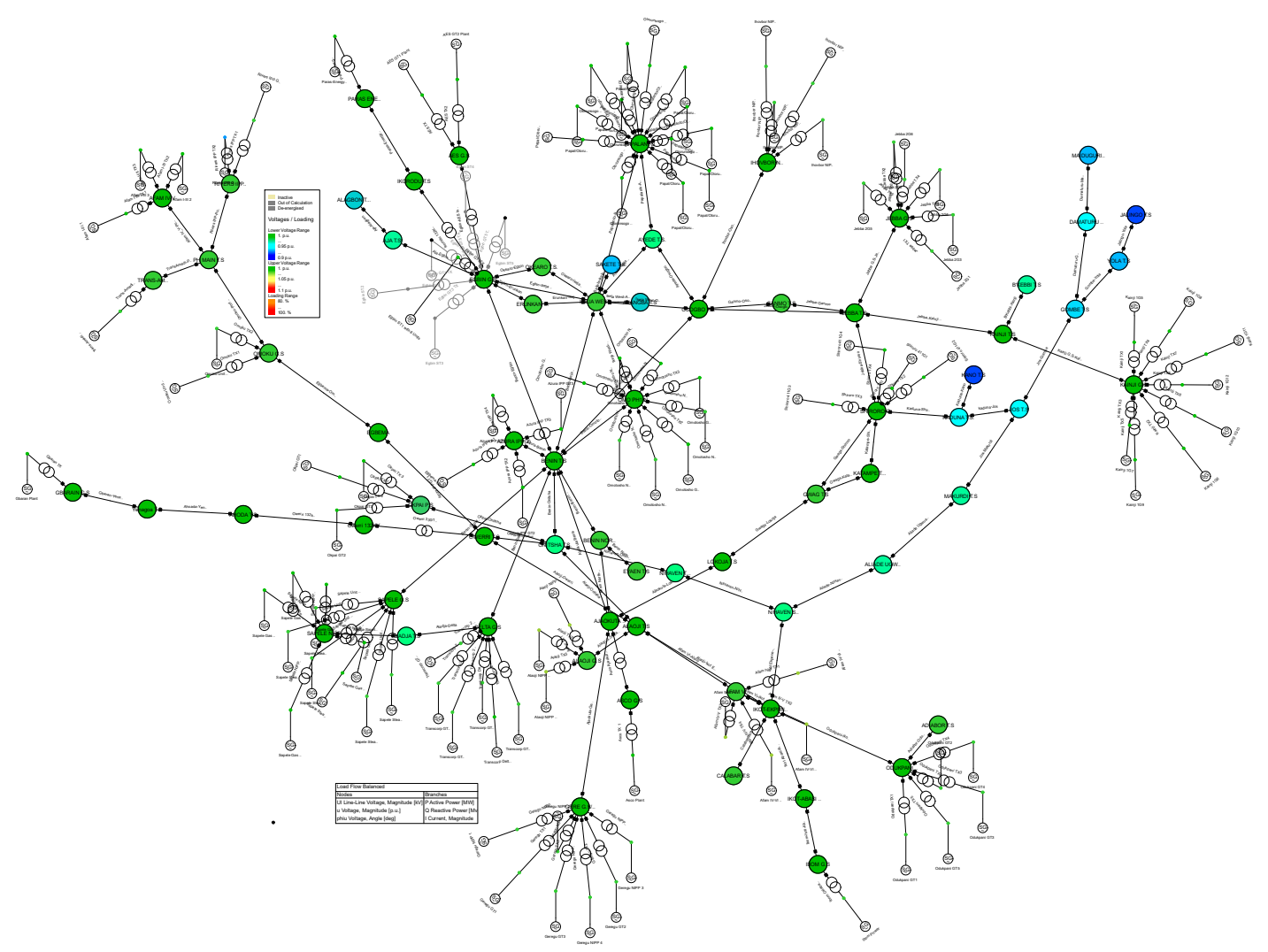

Fig. 1: The Nigerian $330 \mathrm{kV}$ Electrical Power Grid

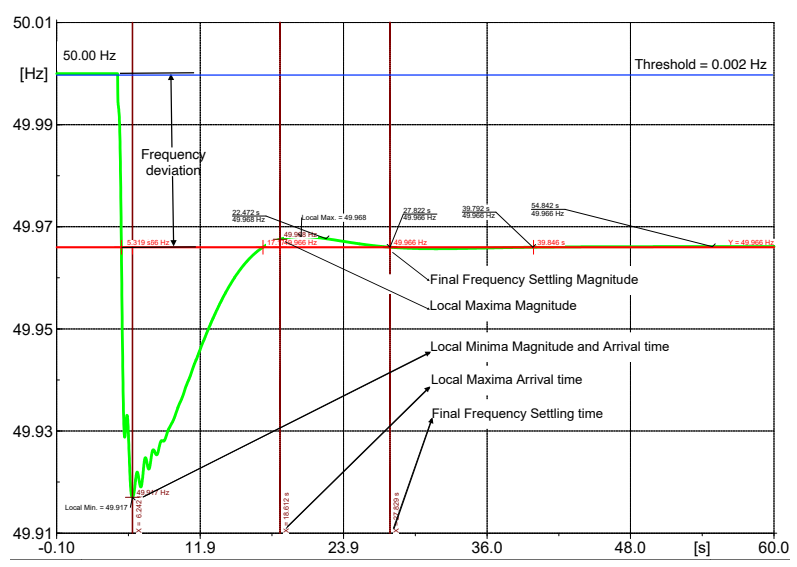

Fig. 2: Green Line: Frequency as a function of time at a node where a Synchronous Machine (SM) Event occurred at $t=$ 5s. Red line: Frequency magnitude at final settling time. Black Arrows: are pointing to the local maxima and minima of the frequency magnitude and arrival times.

\section{Dynamics of MOMEnTARY Reserve}

For a power system to be in a balanced state, the total power generated $(P)$ has to be equal to the sum of the total load and the transmission losses $\left(P^{\mathrm{x}}\right)$. This means that for the system to always remain balanced, any change in the load demand and/ losses would require a balancing change in the generated power $P$. Therefore, the condition $\Delta P>>\Delta P^{\mathrm{x}}$ should always be fulfilled to ensure the security and integrity of the power grids. These dynamic changes result in a permanently changing frequency according to the speed-droop characteristics of power turbines, given by [3]

$$
\frac{\Delta \nu_{i}}{\nu_{0}}=-\sigma_{i} \frac{\Delta P_{i}}{P_{i}^{\mathrm{x}}}
$$

where $\nu_{0}$ is the nominal frequency in Hertz and $\sigma_{i}$ the local droop of the generation characteristics at the $i^{\text {th }}$ generator node, $\sigma_{i}=P_{i}^{\mathrm{x}} / P_{i}$. Thus, any change in frequency resulting from a change in load demand or losses requests a generator response. In this way, if the node is a synchronous generator node, its inertia is expected to slow down the transmission of disturbances to other nodes connected to it, while attempting to damp the frequency oscillations with its momentary reserve according to its droop $\sigma_{i}$ function. However, its ability to do so depends on the amount of momentary reserve that the generator can supply, according to its dead zone speed-droop characteristics setting. Since it is not feasible to inject power equally at every node, we aim to understand the dynamics of these reserves to see how an available momentary reserve at one node influences the frequency dynamics at other nodes as function of their distance from the fault location. A better understanding of this influence in the realistic Nigerian grid 
model would be advantageous for its optimal placement and could improve primary frequency control in real power grids.

To show the influence of geographical distribution of inertia with available momentary reserve, we chose the Nigerian transmission grid as the case study grid. As reported in Section II, we modelled this network in PowerFactory software and calculated the load flow of the network. We considered the voltage dependency of grid loads, and the active power control according to the grid inertia. The effect of provision of momentary reserves by system inertia on the frequency stability of the electrical power networks is studied by varying the aggregated inertia constant $H_{\text {agg }}$ of the entire grid, as defined by [16],

$$
H_{\mathrm{agg}}=\frac{\sum_{i=1}^{N_{S}} H_{i} S_{i}}{S_{n}},
$$

where $S_{i}$ and $H_{i}$ are the rated apparent power and inertia constant of the $i^{t h}$ bus, respectively, $S_{n}=\sum_{i=1}^{N_{S}} S_{i}$ is the total rated power in the grid. Note that $H_{i}$ on non-generator buses are set to zero. The $H_{\text {agg }}$ was varied in the entire grid by multiplying the $S_{i}$ with a common factor. Note that the Nigerian transmission network does not comprise tie-line connections and power transfer [6] [17]. The grid frequency is operated uniformly at $50 \mathrm{~Hz}$ across the entire network.

Here, we choose 11 buses for the investigation. The fault location bus 24, two buses at the same geodesic distance (unweighted), $r=2$ from fault location with no inertia (i.e. buses 8 and 10) and three buses with inertia (i.e. buses 22, 55 , and 57). We again choose two buses at the same geodesic distance (unweighted), $r=7$ with no inertia (i.e. buses 7 and 30 ) and three buses with inertia (i.e. buses 3,28 , and 69 ). The reason is that we want to investigate the effect of momentary reserve both in the vicinity of the reserve (i.e. near to the fault location ) and at far distances from the injection node. We also want to understand how momentary reserve tentatively contributes to damping of disturbances as they propagate along the network. A reference to the network buses numbers is in [18].

Keeping all system parameters, generations, losses and loads constant under undisturbed operations, a synchronous machine outage event (disturbance/contingency) is induced at bus 24 at exactly $t=5 \mathrm{~s}$ of the $90 \mathrm{~s}$ transient electromechanical stability simulation time frame with a $200 \mathrm{~ms}$ switching, enabling the observations on the buses in PowerFactory power simulation software. The change in frequency propagated across the grid is related to the change of power by Eq. (10). From the nodal points described in 2 , we observe at each study case node, the frequency's time of arrival (ToA), as defined when the frequency deviation first reaches a small threshhold of $\delta \nu=0.0001 \mathrm{~Hz}$, as defined in more detail in Ref. [18]. Furthermore we record the time of the first maximum $\left(\right.$ maxima $\left._{t}\right)$ of the transient and its magnitude (maxima $a_{\text {mag }}$ ), the time of the first minimum (minima $t)$ and its magnitude (minima $\left.{ }_{\text {mag }}\right)$. We also observe the final frequency's settling time $\left(\mathrm{FS}_{t}\right)$, its magnitude $\left(\mathrm{FS}_{\mathrm{mag}}\right)$ and frequency deviation
$\left(\operatorname{Dev}_{\text {mag }}\right)$ from the nominal $50 \mathrm{~Hz}$, for each of these nodes. In Tables I-IV we show the observations in milliseconds for the case study nodes, with static network power flow of the Nigerian transmission grid.

TABLE I: Nodal observations with large disturbance and no reserve at fault location given that $H_{\mathrm{agg}}=2 \mathrm{~s}$

\begin{tabular}{lrrrrrrr}
\hline Bus & $\mathrm{r}$ & $\begin{array}{r}\mathrm{ToA} \\
(\mathrm{s})\end{array}$ & $\begin{array}{r}\mathrm{minima}_{t} \\
(\mathrm{~s})\end{array}$ & $\begin{array}{r}\mathrm{minima}_{\mathrm{mag}} \\
(\mathrm{Hz})\end{array}$ & $\begin{array}{r}\mathrm{FS}_{t} \\
(\mathrm{~s})\end{array}$ & $\begin{array}{r}\mathrm{FS}_{\mathrm{mag}} \\
(\mathrm{Hz})\end{array}$ & $\begin{array}{r}\mathrm{Dev}_{\mathrm{mag}} \\
(\mathrm{Hz})\end{array}$ \\
\hline 24 & 0 & 5.012 & 6.522 & 49.596 & 27.200 & 49.829 & 0.171 \\
8 & 2 & 5.012 & 6.522 & 49.596 & 27.201 & 49.829 & 0.171 \\
10 & 2 & 5.012 & 6.522 & 49.596 & 27.200 & 49.829 & 0.171 \\
22 & 2 & 5.013 & 6.282 & 49.596 & 27.195 & 49.829 & 0.171 \\
55 & 2 & 5.013 & 6.532 & 49.596 & 27.201 & 49.829 & 0.171 \\
57 & 2 & 5.012 & 6.532 & 49.597 & 27.196 & 49.829 & 0.171 \\
7 & 7 & 5.013 & 6.372 & 49.598 & 27.205 & 49.829 & 0.171 \\
30 & 7 & 5.013 & 6.322 & 49.600 & 27.206 & 49.829 & 0.171 \\
3 & 7 & 5.013 & 6.422 & 49.590 & 27.185 & 49.829 & 0.171 \\
28 & 7 & 5.013 & 6.942 & 49.596 & 27.184 & 49.829 & 0.171 \\
69 & 7 & 5.013 & 6.352 & 49.592 & 27.186 & 49.829 & 0.171 \\
\hline
\end{tabular}

TABLE II: Nodal observations with large disturbance and large reserve at fault location given that $H_{\text {agg }}=2 \mathrm{~s}$

\begin{tabular}{lrrrrrrr}
\hline Bus & $\mathrm{r}$ & $\begin{array}{r}\text { ToA } \\
(\mathrm{s})\end{array}$ & $\begin{array}{r}\text { minima }_{t} \\
(\mathrm{~s})\end{array}$ & $\begin{array}{r}\text { minima }_{\operatorname{mag}} \\
(\mathrm{Hz})\end{array}$ & $\begin{array}{r}\mathrm{FS}_{t} \\
(\mathrm{~s})\end{array}$ & $\begin{array}{r}\mathrm{FS}_{\mathrm{mag}} \\
(\mathrm{Hz})\end{array}$ & $\begin{array}{r}\mathrm{Dev}_{\operatorname{mag}} \\
(\mathrm{Hz})\end{array}$ \\
\hline 24 & 0 & 5.014 & 6.132 & 49.920 & 25.700 & 49.967 & 0.033 \\
8 & 2 & 5.014 & 6.112 & 49.920 & 25.691 & 49.967 & 0.033 \\
10 & 2 & 5.014 & 6.102 & 49.920 & 25.690 & 49.967 & 0.033 \\
22 & 2 & 5.015 & 6.282 & 49.919 & 25.687 & 49.967 & 0.033 \\
55 & 2 & 5.014 & 6.082 & 49.920 & 25.691 & 49.967 & 0.033 \\
57 & 2 & 5.015 & 6.192 & 49.919 & 25.688 & 49.967 & 0.033 \\
7 & 7 & 5.016 & 6.292 & 49.920 & 25.696 & 49.967 & 0.033 \\
30 & 7 & 5.016 & 6.372 & 49.920 & 25.698 & 49.967 & 0.033 \\
3 & 7 & 5.017 & 6.252 & 49.918 & 25.679 & 49.967 & 0.033 \\
28 & 7 & 5.017 & 6.142 & 49.916 & 25.678 & 49.967 & 0.033 \\
69 & 7 & 5.017 & 6.182 & 49.918 & 25.680 & 49.967 & 0.033 \\
\hline
\end{tabular}

TABLE III: Nodal observations with large disturbance and large reserve at fault location given that $H_{\text {agg }}=6 \mathrm{~s}$

\begin{tabular}{lrrrrrrr}
\hline Bus & $\mathrm{r}$ & $\begin{array}{r}\text { ToA } \\
(\mathrm{s})\end{array}$ & $\begin{array}{r}\text { minima } \\
(\mathrm{s})\end{array}$ & $\begin{array}{r}\text { minima } \\
(\mathrm{Hz})\end{array}$ & $\begin{array}{r}\mathrm{FS}_{t} \\
(\mathrm{~s})\end{array}$ & $\begin{array}{r}\mathrm{FS}_{\mathrm{mag}} \\
(\mathrm{Hz})\end{array}$ & $\begin{array}{r}\text { Dev }_{\operatorname{mag}} \\
(\mathrm{Hz})\end{array}$ \\
\hline 24 & 0 & 5.015 & 9.312 & 49.928 & 29.304 & 49.967 & 0.033 \\
8 & 2 & 5.015 & 9.262 & 49.928 & 29.304 & 49.967 & 0.033 \\
10 & 2 & 5.015 & 9.272 & 49.928 & 29.304 & 49.967 & 0.033 \\
22 & 2 & 5.017 & 8.352 & 49.928 & 29.302 & 49.967 & 0.033 \\
55 & 2 & 5.015 & 9.442 & 49.928 & 29.305 & 49.967 & 0.033 \\
57 & 2 & 5.017 & 9.142 & 49.928 & 29.302 & 49.967 & 0.033 \\
7 & 7 & 5.019 & 9.182 & 49.928 & 29.308 & 49.967 & 0.033 \\
30 & 7 & 5.020 & 8.932 & 49.928 & 29.309 & 49.967 & 0.033 \\
3 & 7 & 5.028 & 8.672 & 49.927 & 29.296 & 49.967 & 0.033 \\
28 & 7 & 5.074 & 8.602 & 49.927 & 29.295 & 49.967 & 0.033 \\
69 & 7 & 5.029 & 8.652 & 49.927 & 29.296 & 49.967 & 0.033 \\
\hline
\end{tabular}

In Table I and with network $H_{\text {agg }}$ at 2 s, a typical behaviour of high renewable energy source injected grids, we observe a high frequency deviation (i.e. $0.171 \mathrm{~Hz}$ ) induced by the large disturbance (of $1320 \mathrm{MW}$ power magnitude outage) at bus 24 fault location. We also observe the disturbance arrival at the fault location first and at the same time as most of its nearest neighbours at $r=2$, but arrived a little later at the distant buses 7, 30, 28 and 69. The frequency dip described by the minima $_{\text {mag }}$ shows the lowest magnitude of frequency deviation before the actions of generator governor-turbine. 
TABLE IV: Nodal observations with large disturbance and large reserve at fault location and an increased reserve at bus 22 given that $H_{\text {agg }}=2 \mathrm{~s}$

\begin{tabular}{lrrrrrrr}
\hline Bus & $\mathrm{r}$ & $\begin{array}{r}\mathrm{ToA} \\
(\mathrm{s})\end{array}$ & $\begin{array}{r}\mathrm{minima}_{t} \\
(\mathrm{~s})\end{array}$ & $\begin{array}{r}\text { minima }_{\mathrm{mag}} \\
(\mathrm{Hz})\end{array}$ & $\begin{array}{r}\mathrm{FS}_{t} \\
(\mathrm{~s})\end{array}$ & $\begin{array}{r}\mathrm{FS}_{\text {mag }} \\
(\mathrm{Hz})\end{array}$ & $\begin{array}{r}\mathrm{Dev}_{\operatorname{mag}} \\
(\mathrm{Hz})\end{array}$ \\
\hline 24 & 0 & 5.014 & 6.612 & 49.920 & 27.645 & 49.967 & 0.033 \\
8 & 2 & 5.014 & 6.122 & 49.921 & 27.647 & 49.967 & 0.033 \\
10 & 2 & 5.014 & 6.612 & 49.920 & 27.645 & 49.967 & 0.033 \\
22 & 2 & 5.015 & 6.352 & 49.919 & 27.641 & 49.967 & 0.033 \\
55 & 2 & 5.014 & 6.582 & 49.920 & 27.647 & 49.967 & 0.033 \\
57 & 2 & 5.015 & 6.152 & 49.921 & 27.642 & 49.967 & 0.033 \\
7 & 7 & 5.016 & 6.342 & 49.921 & 27.653 & 49.967 & 0.033 \\
30 & 7 & 5.016 & 6.452 & 49.921 & 27.655 & 49.967 & 0.033 \\
3 & 7 & 5.017 & 6.282 & 49.919 & 27.630 & 49.967 & 0.033 \\
28 & 7 & 5.017 & 6.142 & 49.917 & 27.629 & 49.967 & 0.033 \\
69 & 7 & 5.017 & 6.252 & 49.919 & 27.631 & 49.967 & 0.033 \\
\hline
\end{tabular}

At $H_{\text {agg }}=2 \mathrm{~s}$ and in comparison with Table I, the Table II shows the observations when the fault location is injected with inertia and large reserve. We observe that there are delays in the frequency ToA at the buses, a reduction in minima m $_{t}$ which corresponds to a reduced frequency dip (i.e. minima $_{\text {mag }}$ ), a shorter final frequency settling time and smaller magnitude and hence, a decrease in the frequency deviation from the nominal value $(0.033 \mathrm{~Hz})$.

In Table III, we kept the network parameters constant and only increased the grid inertia, $H_{\mathrm{agg}}=6 \mathrm{~s}$ using Eq. (11) without changing the active power injections at any network node. We did not observe any further decrement in the frequency deviation or any increment in the frequency final settling magnitude, rather we observe a delay in its time of arrival at the buses with a corresponding increase in its dip and final settling time. This suggest that increasing the $H_{\text {agg }}$ without a corresponding increase in the reserve does not improve the frequency dynamics during contingencies, rather it increases the arrival time of the disturbance while reducing the frequency dip across the network (i.e. improved minima $a_{\text {mag }}$ ). This further delay in ToA also increases the frequency final settling time.

Table IV shows our nodal observations on the case study buses when we injected more power reserve prior to contingency at another bus apart from the fault location and keeping all other system operations parameters constant from observations in Table II. Here the generator's power and reserve at the fault location are higher in magnitude than the injected ones. We observe that the frequency final settling magnitude did not increase, the ToA at the buses remained the same but there is an observable reduction in the frequency dip at some nodes.

In summary, we observe that an increase in the nodal momentary reserve generally delays the travel and arrival of disturbances in a power grid at contingencies. In particular, it improves the frequency magnitude at the local minima point and reduces its final settling time. We found out that the optimal placement of momentary reserve is at the point of contingency as it contributes more in the damping of disturbance across the network more than in any other place.
Since we may not always be able to predict a fault location, the optimal solution would be to place momentary reserve at all buses where resources allow. In this way, the power system could quickly recover most contingencies within few seconds after its occurrence.

Also, we concluded that increasing the grid inertia without a corresponding increase in the magnitude of the reserve could only delay the travel and arrival of disturbances in electrical network but does not reduce the frequency deviation from the nominal value. This result would be important when injecting virtual inertia in the energy transition to renewable schemes.

\section{CONCLUSION}

In this paper, we have studied the dynamics of power system reserves. By realistic numerical experiments we explored how they contribute to the damping of system oscillations at contingencies and thereby work to restore the grid frequency to its nominal value. We have shown that the optimal placement of momentary reserve would be at the fault location, particularly in the case of generator outage events at plant stations. Since this situation could not always be predicted, we suggest placement of reserves in all the nodes where resources permit as this would improve the overall final frequency settling time, frequency dips, and reduce overall frequency deviation from the nominal value, thereby contributing to primary frequency control and reducing the amount of secondary control needed.

Refer to our preprint here [18].

\section{ACKNOWLEDGMENT}

We gratefully acknowledge the support of Bundesministerium für bildung und forschung (BMBF) CoNDyNet-2, FK. 03EK3055D.

\section{REFERENCES}

[1] K. Shi, H. Ye, W. Song, and G. Zhou, "Virtual inertia control strategy in microgrid based on virtual synchronous generator technology," IEEE Access, vol. 6, pp. 27 949-27 957, 2018.

[2] P. Kundur, Power System Stability and Control, 1994.

[3] J. Machowski, J. Bialek, and J. R. Bumby, Power System Dynamics: Stability and Control, 2008.

[4] P. Anderson and A. Fouad, "Power system control and stability," Iowa State University Press, Ames, Iowa, vol. vol. 1, (1977).

[5] DigSILENT GmbH, PowerFactory version 2020 Software Manual, Heinrich-Hertz-Straße 9, 72810, Gomaringen, Germany, 2020.

[6] K. P. Nnoli, "Implementation of a dynamic network model of the Nigerian transmission grid for investigations on power system stability," https://doi.org/10.31224/osf.io/r82zn, 2019.

[7] National Control Center Osogbo, "Transmission data update of the transmission company of Nigeria," 2019.

[8] ENTSO-E, Documentation on Controller Test in Test Grid Configurations, 2013.

[9] G. Rogers, "Demystifying power system oscillations," IEEE Computer Applications in Power, 1996.

[10] IEEE Power Engineering Society, IEEE Guide for Synchronous Generator Modelling Practice and Applications in Power System Stability Analysis. IEEE Press, 2002.

[11] IEEE Power and Energy Society, Dynamic Models for Turbine-Governor in Power System Studies. IEEE Press, 2013.

[12] A. A. Sallam and O. P. Malik, Power System Stability: Modelling, Analysis and Control. IET Power and Energy Series 76, 2015.

[13] A. R. Bergen and D. J. Hill, "A structure preserving model for power system stability analysis," IEEE Transactions on Power Apparatus and Systems, vol. PAS-100, no. 1, pp. 25-35, 1981. 
[14] D. Manik, M. Rohden, H. Ronellenfitsch, X. Zhang, S. Hallerberg, D. Witthaut, and M. Timme, "Network susceptibilities: Theory and applications," Physical Review E 95, 012319, 2017.

[15] K.P. Nnoli, S. Kettemann, "Supplementary material in DataPort," [IEEE DataPort] https://dx.doi.org/10.21227/pjpt-nk47, 2021.

[16] A. Ulbig, T. S. Borsche, and G. Anderson, "Impact of low rotational inertia on power system stability and control," vol. Vol. 14(3), pp. 72907297, 2014.

[17] Nigerian Electricity Regulatory Commission (NERC), The Grid Code for the Nigerian Electricity Transmission System. NERC Press, 2014.

[18] K.P. Nnoli, S. Kettemann, "Spreading of disturbances in realistic models of transmission grids: Dependence on topology, inertia and heterogeneity," [engrarxiv] https://dx.doi.org/10.31224/osf.io/c8awt, 2021. 